\title{
Computational algorithm for stability analysis of distribution networks with distributed generation
}

\author{
Gabriela Rosalee Weigert ${ }^{1^{*}}$ \\ https://orcid.org/0000-0003-2089-602X

\section{Erico Gurski ${ }^{1}$} \\ https://orcid.org/0000-0002-4314-5838
}

\section{Raphael Augusto de Souza Benedito1 \\ https://orcid.org/0000-0001-7654-4205}

${ }^{1}$ Universidade Tecnológica Federal do Paraná (UTFPR), Postgraduate Program in Energy Systems (PPGSE), Department of Electrical Engineering (DAELT), Curitiba, Paraná, Brazil.

Received: 2018.11.05; Accepted: 2019.07.26.

*Correspondence: weigert@alunos.utfpr.edu.br; Tel.: +55-41-33104792 (G.R.W.)

\section{HIGHLIGHTS}

- An algorithm for transient and frequency stability analysis was made.

- Biomass-fed distributed generation was considered.

- A transient stability simulation was made for the on-grid operation.

- A frequency stability simulation was made for the off-grid operation.

\begin{abstract}
With the insertion of distributed generation in distribution networks, security analysis becomes crucial, in islanded operation or when the system is connected to the grid. Dynamic stability analyses are necessary in order to achieve minimum levels of security and reliability. Such analyses are obtained through the solution of the algebraic-differential set of equations that model the distribution grid and their generators, which requires numerical solution methods. Therefore, this paper presents a computational tool for transient stability and large disturbance frequency stability analysis, considering distribution systems with the insertion of biomass-fed distributed generation, which may, or may not, have speed regulation systems. The presented tool may be employed to assess assorted system indexes, such as the severity of disturbances, the quality of a given operating condition, in terms of voltage and frequency operating limits, and the response of both the system and the generators in face of the action of speed regulators.
\end{abstract}

Keywords: distributed generation; biomass; dynamic stability; speed regulator; islanded operation. 


\section{INTRODUCTION}

Distributed Generation (DG) may present itself as a progress path to reliability and flexibility of Electric Power Systems (EPS) [1]. However, the insertion of generation units near the load centers also brings several challenges, whether in a structural aspect, which comes with the variety of generation sources, or in an operational aspect, which embraces security, stability and quality issues [2].

Various energy sources may be used in DG units, such as hydro, photovoltaic and thermal based sources, like natural gas and biomass. In Brazil biomass-based generation is an advantageous option, being flexible in terms of installment area, operative restrictions and even in the raw material alternatives: easily obtained, manipulated and stored [3].

Any organic matter that, given a transformation process, may provide mechanical, thermal or electrical energy may be considered biomass [4]. It may be found as wood, agricultural waste, such as rice hulls and corn cobs, or urban and industrial waste [3]. Biomass source is crucial for the diversification of the Brazilian energy matrix. Its integration with other sources, like hydroelectric, is opportune, once the availability of some materials, such as sugarcane, matches drought periods of some Brazilian regions [4].

In order to generate electrical energy from biomass, it is necessary the use of steam turbines, which may utilize simple or combined-cycle, that includes a heat recovery stage and a variation of the pression in the system [3].

The usage of steam turbines without the heat recovery stage may be justified in the case of smaller machines that don't require high efficiency levels [3]. This premise is adopted in this paper.

From the aspect of operation security, the insertion of DG units may cause dynamic stability issues, such as rotor angle, frequency and bus voltage. All these aspects may attend minimum quality criteria to operate in offline mode or connected to the grid $[5,6]$. Therefore, Dynamic Security Assessment (DSA) is crucial to maintain a normal operating state, providing a service with continuity and quality [7].

The objective of this paper is to present the structure of a dynamic stability computational tool designed for distributing systems with the presence of biomass DG. The proposed algorithm provides studies for transient stability in cases of on-grid and off-grid operation and frequency stability analysis in islanded scenarios.

\section{MATERIAL AND METHODS}

This section presents equation and modelling premises along with the developed computational tool methodology.

\section{System Modelling}

Transient stability analysis for a distribution system with DG insertion is modelled through a Differential-Algebraic (DA) set of equations [1,8,9], presented in (1) and (2). In this system, $g$ is the set of algebraic functions that model the distribution grid instantaneous behavior. The set of state space functions $f$ refer to the mathematical model of Generation Units $(\mathrm{GU})$, representing its slower dynamics.

$$
\begin{gathered}
\frac{d x}{d t}=\dot{x}=f(x, y) \\
0=g(x, y)
\end{gathered}
$$

In this formulation, $x$ represents the state variables vector and $y$ the algebraic one. This set of slow and fast dynamic equations is decomposed and solved separately, alternating between the solution of the algebraic part and the Ordinary Differential Equations (ODE) part.

Thus, in this partitioned solution the algebraic part is calculated first, considering the vector of slow variables $x$ as constant parameters. Obtained the updated values of fast 
variables $y$, the differential variables of the problem $(x)$ are solved, now considering the values of $y$ as constant parameters. This solution is repeated for every point of the trajectory of the analyzed variables.

The algebraic set of equations refers to the distribution grid and is modeled from the Kirchhoff laws and nodal analyses of the studied system. Its solution provides the complex voltages on the buses of the grid [8]. The state space set of equations models GU dynamics and its solution gives access to all the time-domain state variables $[8,10]$.

For the computational tool proposed in this paper, the following mathematical models based on $[3,8,10]$ are enunciated.

Model 1 is composed by the swing equation presented in (3) and (4).

Model 2 is similar to Model 1, however with a non-reheat steam turbine, in (5), a primary speed regulation, in (6), and a secondary speed regulation, in (7).

$$
\begin{gathered}
\dot{\delta}=\Delta \omega \\
\Delta \dot{\omega}=\frac{\left(P_{m 0}+\Delta P_{m}\right)-P_{e}-D \Delta \omega}{M} \\
\Delta \dot{P}_{m}=\frac{\Delta A-\Delta P_{m}}{T_{H P}} \\
\dot{\Delta A}=\left(\frac{-\Delta \omega}{R \cdot \omega_{b}}+\Delta \gamma-\Delta A\right) \frac{1}{T_{G}} \\
\dot{\Delta \gamma}=\frac{-K \Delta \omega}{\omega_{b}}
\end{gathered}
$$

Being $\delta$ the rotor angle variation of the $\mathrm{GU}(\mathrm{rad})$ and $\Delta \omega$ its angular speed variation $(\mathrm{rad} / \mathrm{s}) . M$ is the inertia constant of the machine (pu. $\left.\mathrm{s}^{2} / \mathrm{rad}\right), D$ the damping constant, $\Delta P_{m}$ the mechanical power variation of the turbine $(\mathrm{pu})$ referenced to the equilibrium point. $P_{e}$ is the electrical power transferred by the generator (pu), $\Delta A$ is the governor variation signal and $\Delta \gamma$ the output signal from the secondary regulator. Time constants $T_{H P}$ and $T_{G}$ refer to response rates of the high-pressure state of the turbine and the speed governor, respectively. $R$ is the droop parameter of the machine and $K$ is the integrator gain of the secondary regulator. The synchronous angular speed is represented by $\omega_{b}(\mathrm{rad} / \mathrm{s})$ and is utilized to transform frequency variations from rad/s to pu representation.

\section{Studied System}

The proposed computational tool can be analyzed considering a 33-bus system [11], as depicted in Figure 1.

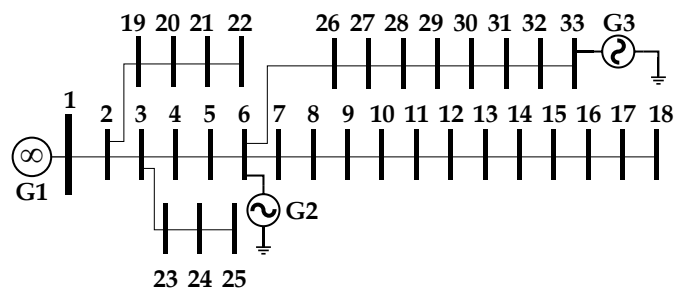

Figure 1. 33-bus system diagram.

Here, the grid is represented by generator G1 as an infinite bus. Units G2 and G3, connected to buses 6 and 33, respectively, are sugarcane based synchronous generators. Both units are modelled with non-reheat steam turbines.

It is, therefore, possible to analyze on-grid scenarios, where the grid is represented by infinite bus 1 , and off-grid scenarios, where line 1-2 is opened and the distribution system operates in islanded mode. 
In terms of definitions proposed in the previous subsection, generator G1 may be represented by Model 1 , considering inertia values of $M$ sufficiently high, so to emulate the behavior of an infinite bus.

Generator G2 is responsible for the load regulation of the islanded operation, being then defined by Model 2. When this GU operates connected to the grid, its regulators are disabled, and its formulation is dictated by Model 1.

Generator G3 does not have any regulatory reserve. It is, Therefore, represented by Model 1.

\section{Proposed Algorithm}

Considering the dynamics of the system operation (on-grid, or off-grid), a computational algorithm for dynamic stability analysis has been developed. The proposed test-system helps to illustrate the different models of GU and its uses. The algorithm general flowchart is presented in Figure 2.

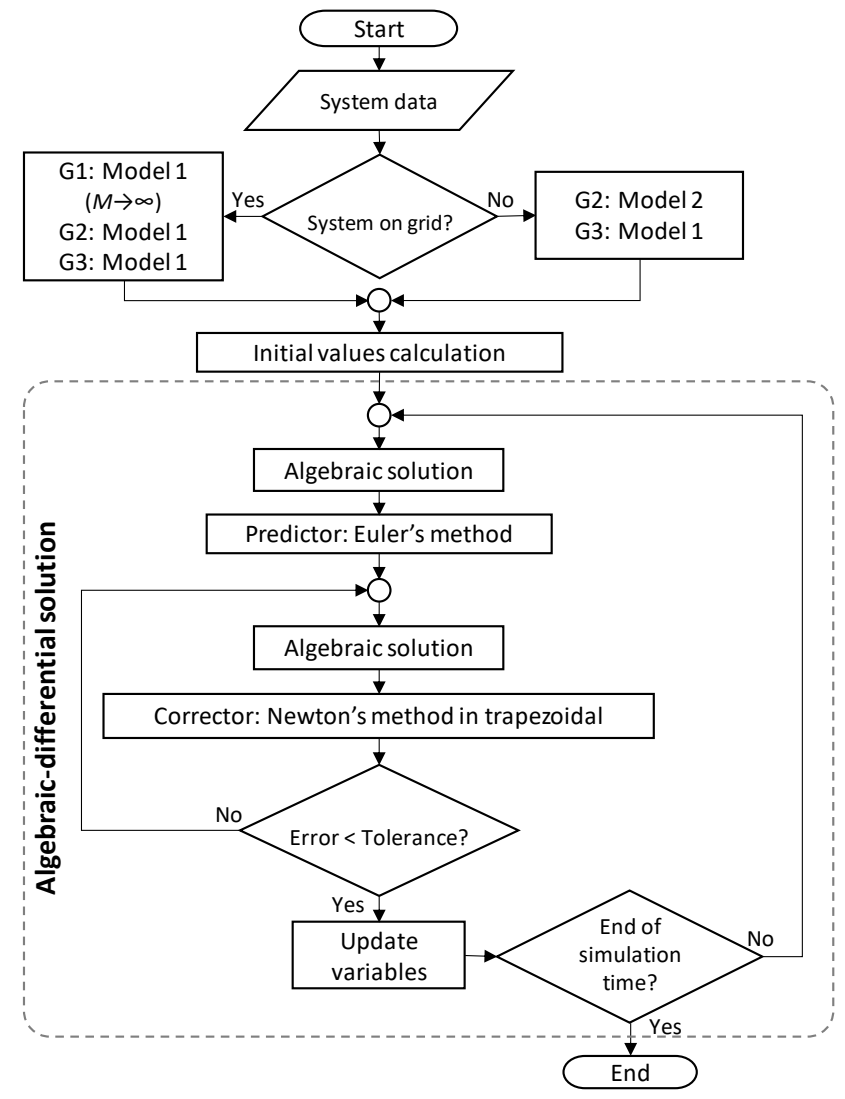

Figure 2. Proposed algorithm flowchart.

The initial points of the simulation come from a power flow study of the system in pre-fault conditions. With these initial values the mathematical model of the GU's of the problem is stablished, such as the initial values of the algebraic and state variables.

Once the system is perturbed, which may occur due to a three-phase fault or a significant step variation of the load, the solution of the trajectory of the analyzed variables begins, solving the algebraic part first and the differential part afterwards.

The numerical method utilized in this solution is the implicit trapezoidal method, executed through a predictor-corrector technique. The predictor values are obtained through explicit Euler's method, while the corrections are made through Newton's method.

After the correction of all the state variables of system, the algorithm proceeds to the next solution, executing the algebraic calculation, then the predictor and corrector phase, repeating this process until the end of the previously specified simulation time. 
The proposed computational tool was developed in MATLAB ${ }^{1}$ code. Its structure and implementation make it possible to replicate the program in other languages, such as $C$ or Fortran.

\section{RESULTS}

Utilizing the proposed methodology, two different simulated scenarios are presented. The first one is a transient stability study and the second one analyzes frequency behavior after large load excursions. Other variables, such as bus voltage, electrical and mechanical power are also analyzed.

Power flow studies previously executed for both scenarios were obtained through Newton Raphson's method. Both cases have stable equilibrium points and do not violate operation limits [5] of bus voltage.

Operation limits were defined in [5]. Frequency after disturbance should be inside the range $59,9 \mathrm{~Hz} \leq f \leq 60,1 \mathrm{~Hz}$. Voltage limits are stratified according to Table 1.

Table 1. Voltage operation limits.

\begin{tabular}{cc}
\hline Classification & $\boldsymbol{V}(\mathrm{pu})$ \\
\hline Adequate & $0.93 \leq V \leq 1.05$ \\
Poor & $0.90 \leq V<0.93$ \\
Critical & $V<0.90$ or $V>1.05$ \\
\hline
\end{tabular}

\section{Transient stability and temporary fault}

The first analyzed scenario considers the whole system operating. Machine parameters are specified in Table 2 and were presented in [12]. In this simulation at instant $t=0$ a temporary three-phase short-circuit is applied on line 1-2, next to infinite bus 1 , which is an extremely severe scenario. All generators in this case are Model 1 type.

Table 2. Generators parameters.

\begin{tabular}{ccccccc}
\hline UG & Bus & $\boldsymbol{X}_{{ }_{\boldsymbol{d}}}^{\prime}(\mathrm{pu})$ & $\boldsymbol{M}\left(\mathrm{pu} . \mathbf{s}^{2} / \mathrm{rad}\right)$ & $\boldsymbol{T}_{\boldsymbol{H P}}(\mathbf{s})$ & $\boldsymbol{T}_{\boldsymbol{G}}(\mathbf{s})$ & $\boldsymbol{K}$ \\
\hline $\mathrm{G} 1$ & 1 & 0.250 & 50000 & - & - & - \\
$\mathrm{G} 2$ & 6 & 4.930 & 0.0003 & 0.3 & 0.2 & 10 \\
$\mathrm{G} 3$ & 33 & 4.930 & 0.0003 & - & - & - \\
\hline
\end{tabular}

Figure 3a presents the oscillography of the rotor angle of all three GU's, considering that the fault is extinct in $100 \mathrm{~ms}$. In this simulation the damping constant considered is $D=$ 0.001 .

In Figure $3 b$ the trajectory of the voltage in some selected buses is presented. After the extinction of the fault and the recovery of the equilibrium in the system, voltage levels do not violate the limits presented in Table 1.

\footnotetext{
1 Academic license number 40685084.
} 


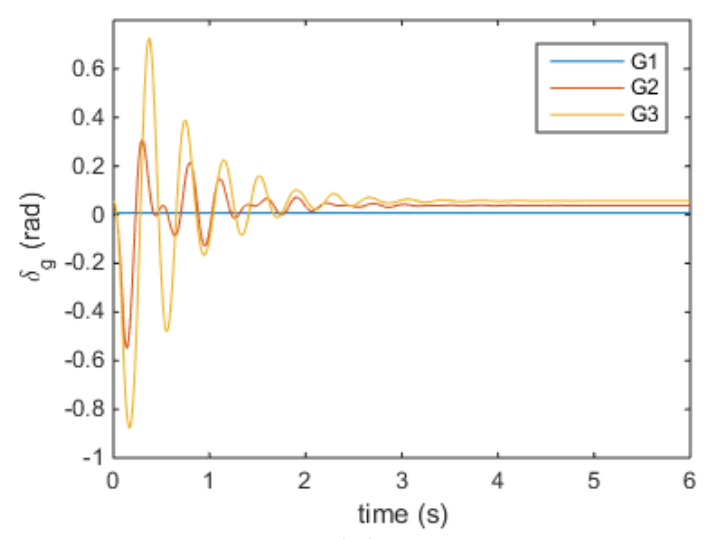

(a)

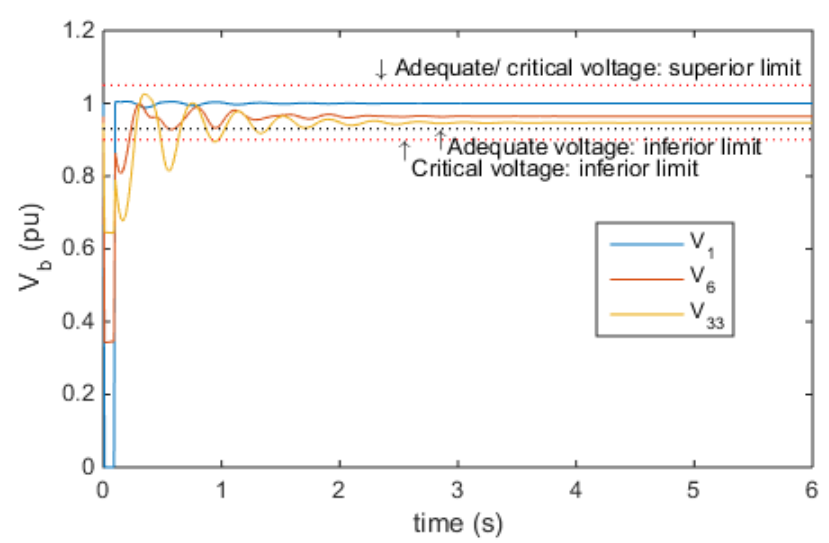

(b)

Figure 3. First scenario system response: (a) Rotor angle oscillography; (b) Voltage level on buses with connected generators.

Analyzing voltage levels at buses with generation it is possible to verify how severe is the fault applied to the system. Bus 33 presents the most oscillatory behavior due to its position. Once the short-circuit is temporary, after its extinction, all buses voltage go back to initial values, maintaining appropriate levels [5].

\section{Frequency stability on off-grid operation}

Considering now a situation where the fault on line 1-2 is permanent, the distribution grid is completely isolated from the main grid and is now supplied only by the DG units. Automatic control demands are satisfied as proposed in [13].

To attend these demands, generator G2 is the new reference of the system, being capable of regulate the frequency, through Model 2 structure. Generator G3 does not have any regulation system, being defined by Model 1 . Now, frequency stability is assessed by inserting load blocks systemically, applying the same step of load at the same instant in all system buses. This kind of scenario may emulate some real situations, such as the behavior of public illumination load.

Damping constant in this example is set in $D=0.005$. The droop parameter is 0.05 and the load variation of $0.1 \mathrm{MW}$ is inserted in all buses at instant $t=1 \mathrm{~s}$.

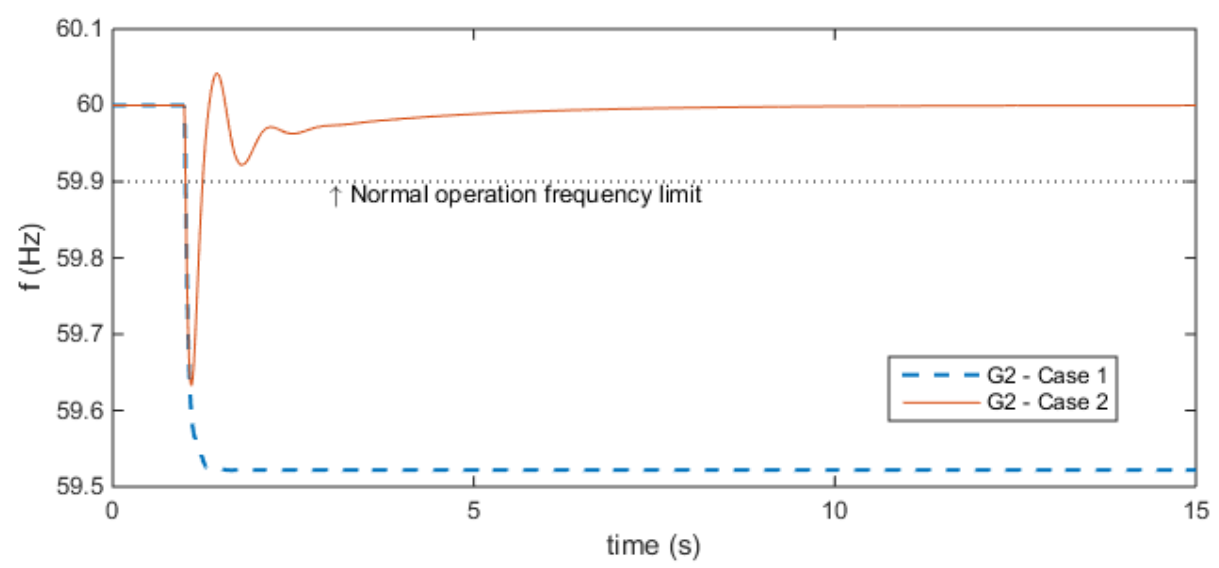

Figure 4. Generator G2 frequency response.

Frequency response of generator G2 is shown in Figure 4. A comparison between Case 1 (no load control at the system) and Case 2 (primary and secondary speed regulation are made by G2) is established. Response of generator G3 is not shown due to its superposition with G2's.

When frequency limits are trespassed, the occurrence of a disturbance is characterized. In terms of transitory limits, there are no violations [5]. The reestablishment of frequency 
levels occurs in a fast way due to its fast turbine and governor time constants and the low characteristic inertia of this type of machines.

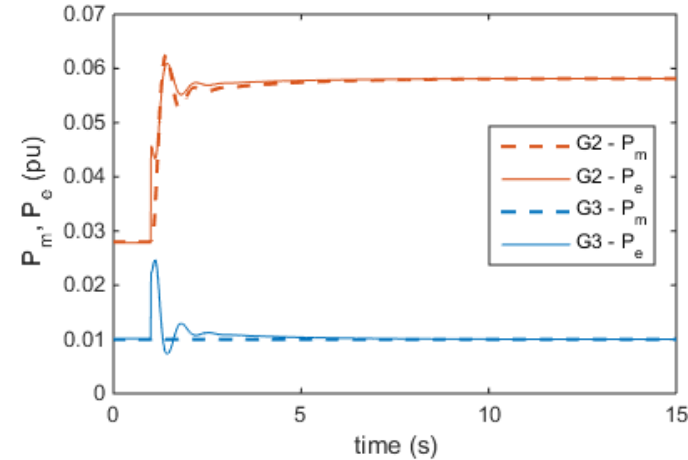

(a)

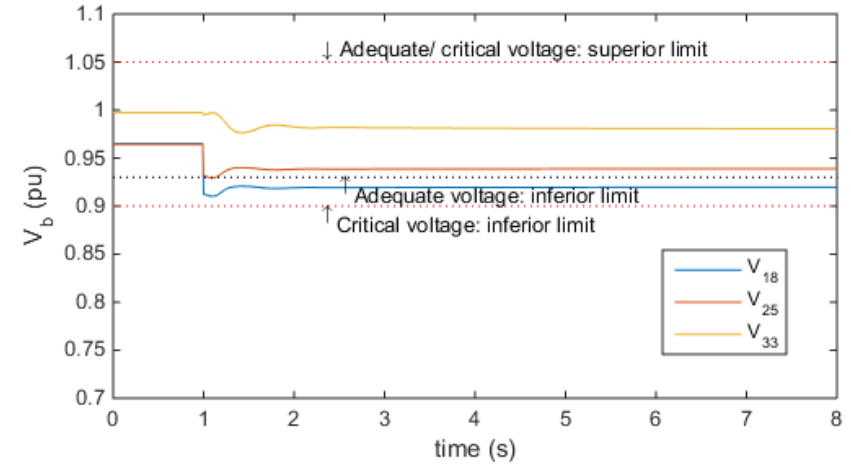

(b)

Figure 5. Second scenario system response: (a) Mechanical and electrical power; (b) Voltage level on critical buses.

The load regulation supplied by generator G2 may be observed through mechanical power curves, in Figure 5a, which show how generator $\mathrm{G} 2$ delivers the necessary power to equilibrate the power balance again in the system.

Analyzing critical buses' levels of voltage, in Figure 5b, it is possible to verify how the systemic load insertion affects bus voltage, mainly the ones at the end of long branches. Although there is no violation of inferior critical limits, the voltage on bus 18 violates the minimum adequate level of voltage. This kind of behavior is expected, once the modelling of the network equipment and generators for this algorithm does not include voltage regulators.

\section{DISCUSSION}

This paper presented a transient and frequency stability computational simulation tool applicable to distribution systems with the insertion of distributed generation. Considering the on-grid and off-grid setups, this tool relies on a partitioned algebraic-differential solution and sees all the buses of the system and its parameters, differently from methods that employ topology reductions and simplifications.

The great contribution of this algorithm is the possibility of DSA in a flexible way: considering the system connected to a main grid; or disconnected, adapting the generators models to each situation. This tool can also be used in operation planning and electro-energetic expansion of distribution systems, once it facilitates the calculation of load variations, bus voltage, rotor angle and frequency on time-domain, as shown in this paper results.

Future research may include more aspects of the network, analyzing system stability with the addition of other equipment dynamics, such as protection and voltage regulation, and disturbances with different behaviors.

\section{REFERENCES}

1. Lora, E. E. S.; Addad, J. Geração distribuída: aspectos tecnológicos, ambientais e institucionais. Interciência: Rio de Janeiro, Brazil, 2006.

2. Bollen, M. H. J.; Hassan, F. Integration of distributed generation in the power system. Wiley-IEEE Press: USA, 2011.

3. Lora, E. E. S.; Nascimento, M. A. R. do. Geração termelétrica: planejamento, projeto e operação. Interciência: Rio de Janeiro, 2004; Volume 2.

4. Agência Nacional de Energia Elétrica - Aneel. Atlas de energia elétrica do Brasil, 3a ed. Aneel: Brasília, 2008. 
5. Agência Nacional de Energia Elétrica - Aneel. Procedimentos de Distribuição de Energia Elétrica no Sistema Elétrico Nacional - PRODIST: Módulo 8 - Qualidade da Energia Elétrica. January 2018.

6. IEEE Standard for Interconnection and Interoperability of Distributed Energy Resources with Associated Electric Power Systems Interfaces, IEEE Std 1547-2018, 2018.

7. Kundur, P. et al.. Definition and Classification of Power System Stability, IEEE Transactions on Power Systems 2004, Volume 19, no. 2, pp. 1387 - 1401.

8. Kundur, P. Power System Stability and Control. McGraw Hill: USA, 1994.

9. Cutsem, T. V.; Vournas, C. Voltage Stability of Electric Power Systems. Kluwer: Norwell, 1998.

10. Vieira Filho, X. Operação de sistemas de potência com controle automático de geração. Eletrobrás: Rio de Janeiro, 1984.

11. Baran, M. E.; Wu, F. F. Network reconfiguration in distribution systems for loss reduction and load balancing, IEEE Transactions on Power Delivery 1989, Volume 4, no. 2, pp. $1401-1407$.

12. AVK. Technical Data Sheet for AvK-Alternators: FM 7.3-5. Datasheet DIG 156 o/8, 2013.

13. Agência Nacional de Energia Elétrica - Aneel. Procedimentos de Distribuição de Energia Elétrica no Sistema Elétrico Nacional - PRODIST: Módulo 4 - Procedimentos Operativos do Sistema de Distribuição. January 2010. 